\title{
Thermographic Assessment of Reperfusion Profile Following Using a Tourniquet in Total Knee Arthroplasty: A Prospective Observational Study
}

\author{
Mohammed Alisi (iD' \\ Jihad Al-Ajlouni (iD) \\ Mohammed Kareem Ibsais ${ }^{2}$ \\ Zeinab Obeid ${ }^{2}$ \\ Yazan Hammad (1D) \\ Ahmad Alelaumi' \\ Munther Al-Saber' \\ Odai Abuasbeh' \\ Feras Abuhajleh' \\ 'Department of Special Surgery, Division \\ of Orthopaedics, School of Medicine, The \\ University of Jordan, Amman, Jordan; \\ ${ }^{2}$ School of Medicine, The University of \\ Jordan, Amman, Jordan
}

Background: Infrared thermal imaging is a non-invasive technique capable of detecting changes in temperature that could ultimately signify changes in blood supply. Flir One is a smartphone-based thermal camera, working by a downloadable application, capable of detecting the limb temperature through a non-contact method using infrared thermography technology. Using the Flir One camera, we will assess the lower limb reperfusion profile following the tourniquet release post total knee arthroplasty (TKA).

Methods: A prospective study included 46 patients who underwent primary TKA. We used the (Flir One Gen 3) thermographic camera to capture images at ankle joint preoperatively, and at 1, 10, and 20 minutes post tourniquet release on operation side. The contralateral ankle stands as control. Results: The mean preoperative temperature (in Celsius) of ankle control side and operated side were $33.03(\mathrm{SD}=1.65)$ and $33.26(\mathrm{SD}=1.42)$, respectively. The mean ankle temperature on operation side was $19.73(\mathrm{SD}=2.85), 30.49(\mathrm{SD}=2)$, and $32.43(\mathrm{SD}=1.31)$ at 1,10 , and 20 minutes post tourniquet release, respectively, while the control side showed a mean temperature of $32.85(\mathrm{SD}=1.42), 32.84(\mathrm{SD}=0.91)$, and $33.15(\mathrm{SD}=0.95)$ at the same time intervals. There was a significant statistical difference between both ankle temperatures at 1 and 10 minutes $(\mathrm{P}=0.00$ for each time). At 20 minutes, 37 ankles $(80.4 \%)$ at operation side reached a temperature level similar but below the level of control side; however, the difference was not significant $(\mathrm{P}=0.692)$.

Conclusion: Infrared thermography using the smartphone-connected camera is a simple, non-invasive, feasible, and reliable technology. It provides an objective measure to assess the perfusion status of the limbs. In TKA, the distal limb will reach full reperfusion status after approximately 20 minutes of tourniquet release.

Keywords: thermography, thermal imaging, perfusion, total knee arthroplasty, temperature, infrared

\section{Introduction}

Dynamic blood flow to the skin is responsible for the regulation of thermal homeostasis. Vasodilation of blood vessels activated by sympathetic cholinergic nerves increases the skin's temperature, while a decrease occurs in vasoconstriction. ${ }^{1,2}$ Thermography has been verified for the evaluation of normal skin temperatures in the population. ${ }^{3,4}$ Infrared thermographic-based imaging is a non-invasive technique capable of detecting changes in temperature that could ultimately signify changes in blood supply., 5

This study aims to investigate the revascularization profile following using a tourniquet in total knee arthroplasty (TKA) by detecting cutaneous temperature
Correspondence: Jihad Al-Ajlouni School of Medicine, The University of Jordan, Queen Rania Al Abdullah St 266 Al Jubaiha, Amman, II942, Jordan Tel +962799060817

Email jajlouni@hotmail.com 
changes using smartphone-based thermographic imaging. The focus will be on the time needed to reestablish vascularization immediately after the release of tourniquet. We hypothesize that the limb returns to the full perfusion status (similar to the contralateral side) immediately after the release of tourniquet after total knee replacement surgery.

To the best of our knowledge, no previous studies reported the revascularization profile by using infrared thermography following using a tourniquet in TKA.

\section{Methods}

This prospective cohort study has investigated patients undergoing unilateral primary TKA. The sample was selected randomly to include all patients who were planned for elective TKA over 6 months at an academic institution. We excluded patients with the following conditions: inflammatory arthritis, revision arthroplasty, symptoms/signs of peripheral vascular disease (such as claudication, delayed capillary refill, shiny skin, loss of hair, ulcers, and calcified vessels on knee radiographs), previous surgery on the control side, and who had skin disorder on lower limbs.
We obtained approval for this study from the institutional review board at Jordan University Hospital. All participants signed an informed consent to be included in this study.

\section{Equipment (Figure I)}

The thermal images were taken using a Flir One thermal camera operated by a smartphone device. The camera is physically connected to a smartphone by USB-C input and is used to capture still images of the joints (Figure 1A). The smartphone used is a Nokia mobile phone with the Android version of Flir One Gen 3 camera using a downloadable application (Figure 1B).

Flir One Gen 3 thermal camera is capable of detecting infrared energy through a non-contact method. It converts the infrared readings into an electronic signal, which is then interpreted into numerical calculations of temperature in Fahrenheit. The resolution of the camera is 0.1 degrees Fahrenheit. The camera operates under temperatures between negative 4- and 140-degrees Fahrenheit. The thermal resolution of the device is $17 \mu \mathrm{m}$ with an $8-14 \mu \mathrm{m}$ spectral range.

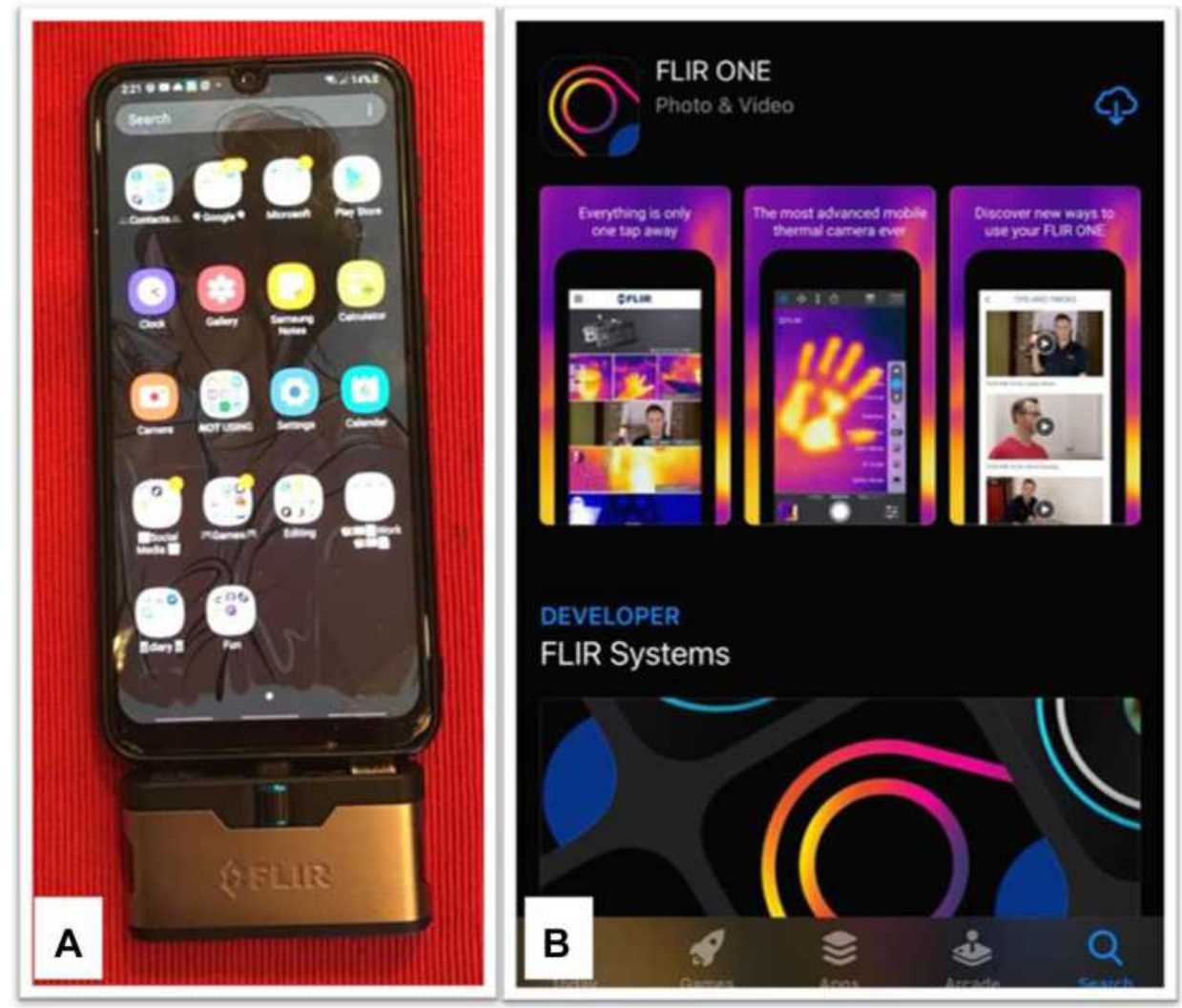

Figure I Showing the Flir One thermal camera connected to the smartphone (A) and operated using an application available on the Google Play store (B). 


\section{Measurement}

The study employs a thermographic camera to capture images at the ankle joint (Figure 2). Imaging of the ankle taken before application of the tourniquet and after the release of the tourniquet at 1, 10, and 20 minutes. The contralateral limb stands as the control for each time point. These images serve to detect changes in blood supply immediately after the release of the tourniquet. The camera was mounted $20-30 \mathrm{~cm}$ above the surface of the skin of the medial malleolus. The images have been taken in the same operating room and recovery room for all patients. The average environmental temperature for the operating and recovery room was $21.6(\mathrm{SD}=1.4)$ and $23.5(\mathrm{SD}=2.6)$ Celsius respectively, while the average humidity was $38.2 \%$ $(\mathrm{SD}=8.6)$ and $34.9 \%(\mathrm{SD}=5.9)$ respectively.

The tourniquet was placed around the thigh $10-15 \mathrm{~cm}$ above the surgical site for an average duration of 1.5 hours. The tourniquet pressure was $150 \mathrm{mmHg}$ above systolic blood pressure but not exceeding $350 \mathrm{mmHg}$ overall.

All surgeries were done by one expert orthopaedic surgeon by the same technique and using identical prosthesis type (Zimmer Biomet) according to the surgeon's preference. The surgical approach was a medial parapatellar for all patients. The mode of anesthesia was either general or spinal anesthesia depending on the needs of each patient.

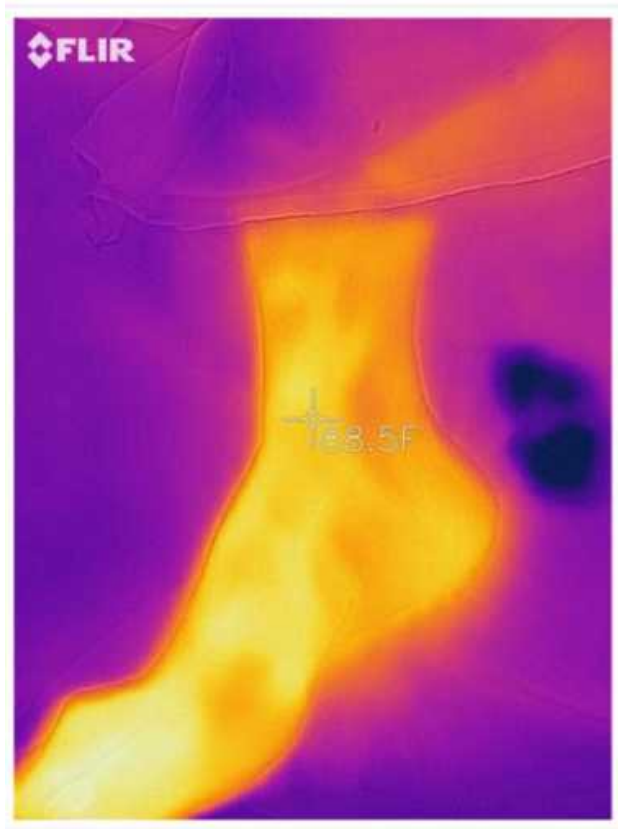

At the end of surgery, in the operating room, we captured the images after 1 minute of releasing the tourniquet, while the patient lying supine.

Postoperatively, in the recovery room, the images were captured at 10 and 20 minutes while the patient in a supine position with neutral leg height on the bed without elevation. All patients were covered with the usual blankets without using any forced-air warming machines.

\section{Statistical Analysis}

We used PASW statistics 18 (IBM-USA) for data analysis. The Shapiro-Wilk test was used to check the normality of the data. Analysis of variance (ANOVA) and post hoc multiple comparisons Bonferroni tests were used to compare the means of our values. P-value $<0.05$ was considered statistically significant. We converted the temperature degree from Fahrenheit to Celsius for easy understanding in our community.

\section{Results}

A total of 46 patients were involved in the study (43 females and 3 males). The mean age was 64.46 years $(\mathrm{SD}=4.39)$. Twenty-five patients underwent left TKA and 21 had right TKA. Table 1 illustrates the demographic data of the included patients.

The mean preoperative ankle temperature (in Celsius) on the control side and operation side was 33.03

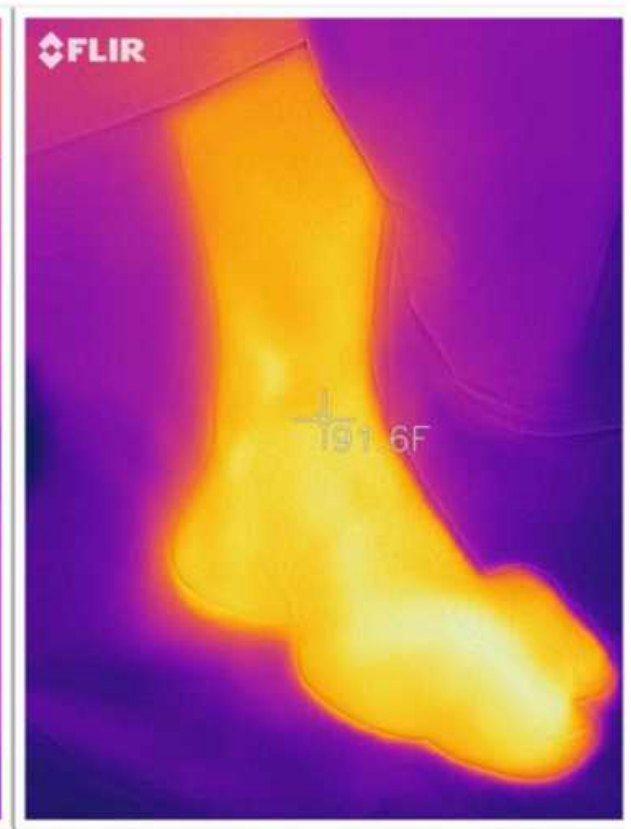

Figure 2 Showing the thermographic images captured by smartphone-connected Flir One camera measuring the ankle joint temperature. 
Table I Illustrates the Demographic Data of the Included Patients

\begin{tabular}{|l|c|c|}
\hline Variables & Mean & Standard Deviation \\
\hline Age & 64.46 & 4.39 \\
Body mass index & 34.3 & 4.14 \\
Operation time (min) & 101 & 19 \\
\hline & Count & Percentage \\
\hline Side & 25 left side & $54.3 \%$ \\
Gender & 43 females & $93.4 \%$ \\
Diabetes mellites & 18 & $39.1 \%$ \\
Hypertension & 33 & $71.7 \%$ \\
Ischemic heart disease & 4 & $8.7 \%$ \\
Chronic kidney disease & 1 & $2.2 \%$ \\
Smoker & 14 & $30.4 \%$ \\
Spinal anesthesia & 42 & $91.3 \%$ \\
General anesthesia & 4 & $8.7 \%$ \\
\hline
\end{tabular}

$(\mathrm{SD}=1.65)$ and $33.26(\mathrm{SD}=1.42)$ respectively. The difference was not statistically significant between both ankles at this point $(\mathrm{P}=1.00)$. The mean postoperative temperature of the ankle on the operation side was 19.73 $(\mathrm{SD}=2.85), 30.49 \quad(\mathrm{SD}=2)$, and $32.43 \quad(\mathrm{SD}=1.31)$ at 1 minute, 10 minutes, and 20 minutes post-release of tourniquet, respectively. On the other side, the mean temperature of the ankle on the control side was 32.85 $(\mathrm{SD}=1.42), 32.84(\mathrm{SD}=0.91)$, and $33.15(\mathrm{SD}=0.95)$ at the same time intervals (Figure 3).

The difference was statistically significant between both ankle temperature values at 1 minute and 10 minutes post-release of the tourniquet $(\mathrm{P}=0.000$ for each time interval). At 20 minutes post-release of the tourniquet, 37 ankles (80.4\%) at the operation side reached a temperature level similar but below the level of the control side; however, the difference was not significant $(\mathrm{P}=0.692)$ (Figure 4).

\section{Discussion}

Thermal imaging is a modern technology that is becoming increasingly popular in medicine. It has been utilized for detection of changes in skin temperature in the form of inflammation, neovascularization, and vasoconstrictive phenomena as well as direct thermographic changes that signify blood supply. Thermal imaging has been used in several clinical and operative settings. These include but are not limited to detecting the response to treatment in inflammatory arthritis, diagnosis of the complex regional pain syndrome, monitoring the surgical procedures in open-heart and brain tumor surgeries, and fever

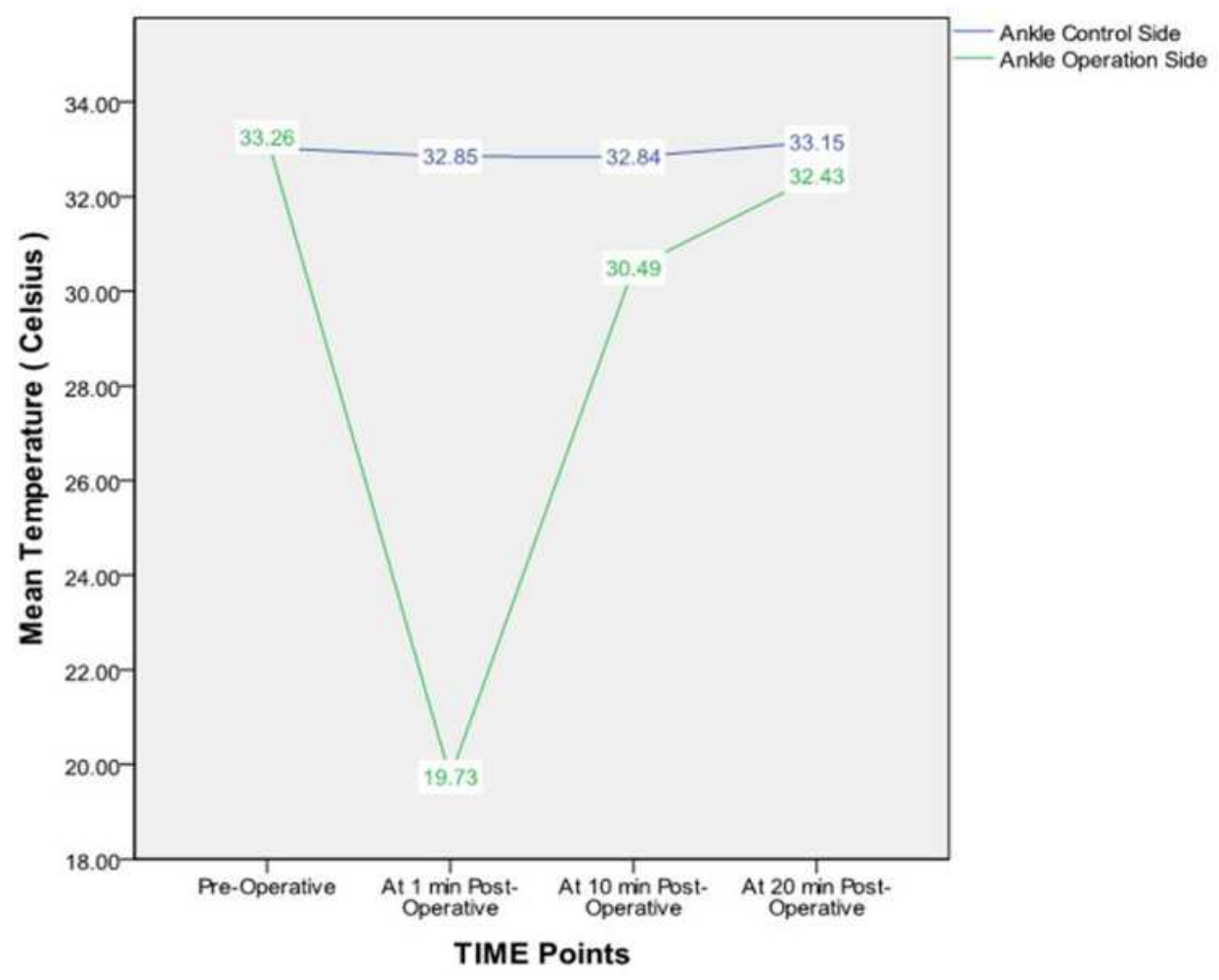

Figure 3 Showing the mean ankle temperature on the operation (green) and control (blue) sides at different times. 


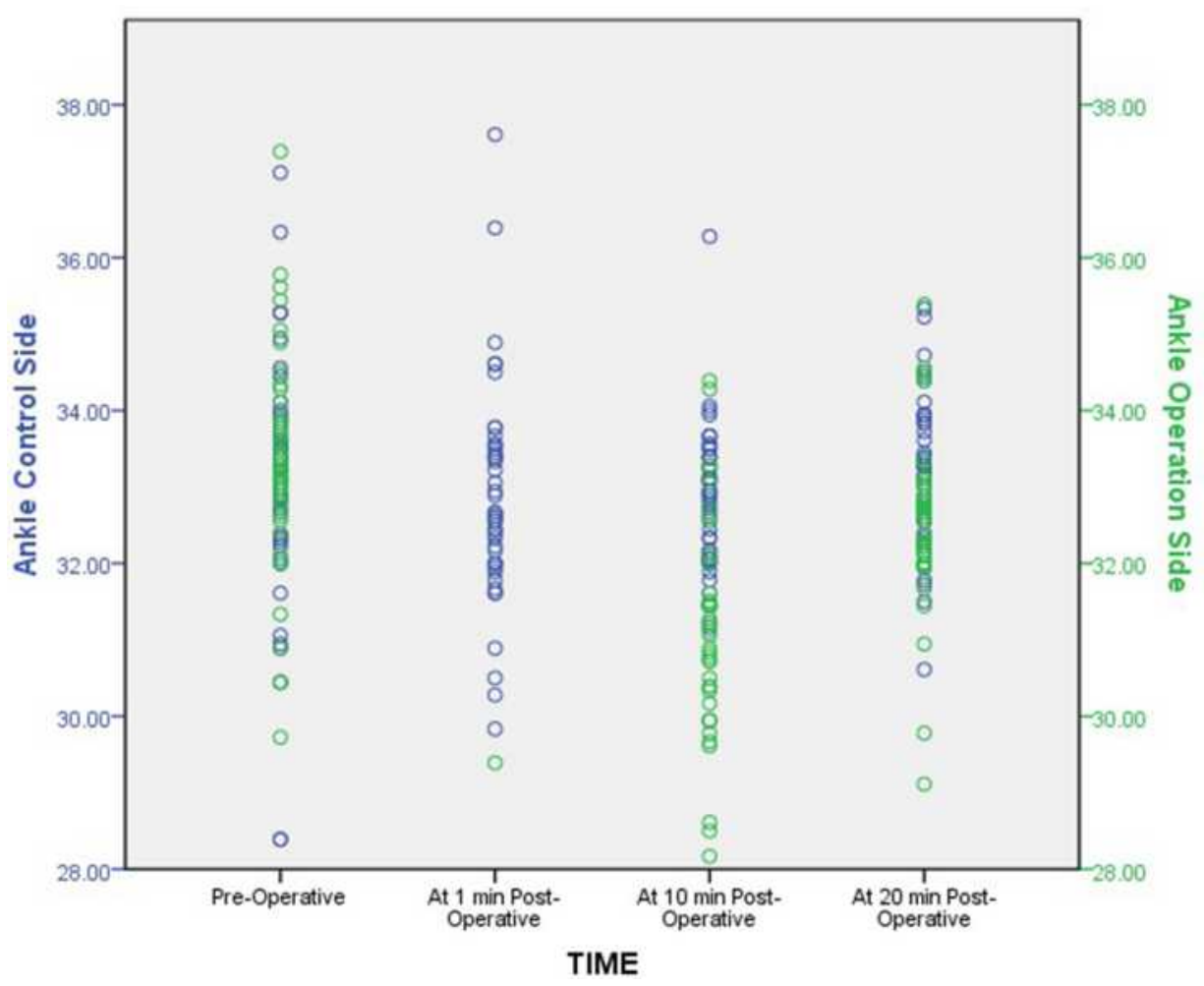

Figure 4 Showing the temperature of the ankle on the operation side (green) as it getting closer to the control (blue) side at 20 minutes post-release of the tourniquet.

screening. ${ }^{7-9}$ Furthermore, thermal imaging is considered an efficient tool for assessment of revascularization in multiple entities such as diabetic foot, arteriovenous fistula, Raynaud's phenomenon, kidney transplantation, and skin grafts. ${ }^{10,11}$

In total knee arthroplasty, several studies have been reported the use of thermal imaging for assessment of local warmth, anterior knee pain, and prosthetic joint infection postoperatively. ${ }^{12-16}$ Glehr et $\mathrm{al}^{12}$ concluded that thermography can be considered as a rapid and inexpensive technology to assess knee pain and inflammation post total knee arthroplasty. In more than one article, Romanò et al ${ }^{13,14}$ studied the effectiveness of thermography for diagnosing periprosthetic infections post TKA. They reported that thermal imaging is a reliable option to diagnose periprosthetic knee infection.

Paton and $\mathrm{Neal}^{17}$ and Patil et $\mathrm{al}^{18}$ studied the effect of TKA on distal limb vascularity. They assessed the operated limb by Doppler ultrasonography and concluded that TKA does not affect the blood flow in the lower limb postoperatively.

Our study assesses and profiles the reestablishment of blood supply in the distal limb in the immediate postoperative period after the release of tourniquet. We assessed revascularization of the limb using thermal imaging by detecting the change in the ankle temperature at regular intervals post tourniquet release.

Infrared cameras can be classified into 3 categories according to their spectral range; Short- Wavelength Infrared (SWIR: 1.4-3 $\mu \mathrm{m}$ ), Mid-Wavelength Infrared (MWIR: 3-8 $\mu \mathrm{m}$ ), and Long-Wavelength Infrared (LWIR: $8-15 \mu \mathrm{m})$. The SWIR cameras have limited use due to poor thermal radiation within their spectral range. The MWIR cameras have been widely used within the field of thermography, but they are very expensive and usually available in specialized research centers. Although the LWIR cameras have lower quality images, it is less expensive by $200 \mathrm{X}$ than the MWIR cameras. Razani et $\mathrm{al}^{19}$ validated the use of the cheap LWIR cell phones-connected cameras for thermographic assessment of biological materials. Furthermore, recent studies demonstrated the validity and applicability of using the cell phones-connected thermal cameras for different clinical implications. ${ }^{20-23}$

FLIR ONE is a LWIR smartphone-connected thermal camera. It is a light hand-held device, easy to use, noninvasive, cheap, reliable, and an objective tool that provides an immediate reading for skin temperature and reflects the perfusion status of the limb. ${ }^{24}$ 
A tourniquet will temporarily halt the blood supply as a mean of avoiding excess intraoperative blood loss and providing a clean surgical field. The blood supply to the knee is cut off intraoperatively by raising the tourniquet cuff pressure above the systolic arterial pressure and reestablished after wound closure. Hence, reperfusion is expected to occur in the immediate postoperative period. This study has profiled postoperative reperfusion in patients who underwent TKA after the release of the tourniquet to test this hypothesis.

The results of this study showed that temperature at the ankle joint of the operated side was significantly lower than the control side immediately (at 1 minute) and at 10 minutes after the release of the tourniquet. However, the temperature of the ankle joint of the operated side increased gradually till it reaches a similar level of the control limb at 20 minutes post-release of the tourniquet. These results refute the hypothesis of the immediate reperfusion of the distal limb after the release of the tourniquet in patients who underwent primary TKA. Of note, none of our patients have documented peripheral vascular disease or calcified vessels on knee radiographs.

The clinical implications of this study include that thermal imaging can be used as a tool to ensure the postoperative lower limb perfusion status in patients with severe vascular disease or who have impalpable dorsalis pedis pulsation. Besides, we can use thermal imaging as an objective tool in the operative room to determine the tourniquet pressure at which the blood supply of the lower limb is sufficiently halted. Mu et $\mathrm{al}^{25}$ reported the use of Doppler ultrasound to detect tourniquet pressure in the lower limb.

Limitations of this study include its observational nature and a relatively small number of patients. Furthermore, we suggest further studies to validate the use of thermal imaging to detect postoperative reperfusion in patients with severe vascular disease.

\section{Conclusion}

Infrared thermal imaging using the smartphone-connected camera is a simple, non-invasive, and reliable technology to assess the perfusion of the limbs. It is feasible to be used in emergency situations and offering a wide range of clinical implications.

This study emphasizes the practicality of using the low-cost thermal camera as an objective tool for detecting the postoperative limb perfusion status. In TKA, the distal limb will reach full reperfusion status after approximately 20 minutes of tourniquet release.
We advise the researchers to investigate the applicability of this technology for further clinical situations.

\section{Ethics and Consent}

The authors certify that this research was approved by institutional review board at Jordan University Hospital and this study complied with the Declaration of Helsinki. We obtained informed consent from all included patients to have their data.

\section{Funding}

This research received no specific grant from any funding agency in the public, commercial, or not-for-profit sectors.

\section{Disclosure}

The authors reported no conflicts of interest for this work.

\section{References}

1. Christen S, Delachaux A, Dischl B, et al. Dose-dependent vasodilatory effects of acetylcholine and local warming on skin microcirculation. J Cardiovasc Pharmacol. 2004;44(6):659-664. doi:10.1097/00005344-200412000-00006

2. Wong BJ, Hollowed CG. Current concepts of active vasodilation in human skin. Temperature (Austin). 2016;4(1):41-59. doi:10.1080/ 23328940.2016.1200203

3. Alfieri FM, Battistella LR. Body temperature of healthy men evaluated by thermography: a study of reproducibility. Technol Health Care. 2018;26(3):559-564. doi:10.3233/THC-171164

4. Gatt A, Formosa C, Cassar K, et al. Thermographic patterns of the upper and lower limbs: baseline data. Int $J$ Vasc Med. 2015;2015:831369. doi:10.1155/2015/831369

5. Sagaidachnyi AA, Fomin AV, Usanov DA, Skripal AV. Thermography-based blood flow imaging in human skin of the hands and feet: a spectral filtering approach. Physiol Meas. 2017;38 (2):272-288. doi:10.1088/1361-6579/aa4eaf

6. Shilco P, Roitblat Y, Buchris N, et al. Normative surface skin temperature changes due to blood redistribution: a prospective study. J Therm Biol. 2019;80:82-88. doi:10.1016/j.jtherbio.2019. 01.009

7. Fokam D, Lehmann C. Clinical assessment of arthritic knee pain by infrared thermography. J Basic Clin Physiol Pharmacol. 2018;30(3). doi:10.1515/jbcpp-2017-0218

8. Ring EF, Ammer K. Infrared thermal imaging in medicine. Physiol Meas. 2012;33(3):R33-R46. doi:10.1088/0967-3334/33/ 3/R33

9. Sadeghi-Goughari M, Mojra A. Intraoperative thermal imaging of brain tumors using a haptic-thermal robot with application in minimally invasive neurosurgery. Appl Therm Eng. 2015;91:600-610. doi:10.1016/j.applthermaleng.2015.08.032

10. Fernandez N, Lorenzo A, Chua M, Koyle MA, Farhat W, Matava C. Real-time kidney graft perfusion monitoring using infrared imaging during pediatric kidney transplantation. J Pediatr Urol. 2019;15(3):222.e1-222.e7. doi:10.1016/j.jpurol. 2019.03.016

11. van Doremalen RFM, van Netten JJ, van Baal JG, VollenbroekHutten MMR, van der Heijden F. Validation of low-cost smartphone-based thermal camera for diabetic foot assessment. Diabetes Res Clin Pract. 2019;149:132-139. doi:10.1016/j. diabres.2019.01.032 
12. Glehr M, Stibor A, Sadoghi P, et al. Thermal imaging as a noninvasive diagnostic tool for anterior knee pain following implantation of artificial knee joints. Int J Thermodyn. 2011;14 (2):71-78. doi:10.5541/ijot.334

13. Romanò CL, Logoluso N, Dell'Oro F, Elia A, Drago L. Telethermographic findings after uncomplicated and septic total knee replacement. Knee. 2012;19(3):193-197. doi:10.1016/j. knee.2011.02.012

14. Romanò CL, D’Anchise R, Calamita M, Manzi G, Romanò D, Sansone V. Value of digital telethermography for the diagnosis of septic knee prosthesis: a prospective cohort study. BMC Musculoskelet Disord. 2013;14(1):7. doi:10.1186/1471-2474-14-7

15. Haidar SG, Charity RM, Bassi RS, Nicolai P, Singh BK. Knee skin temperature following uncomplicated total knee replacement. Knee. 2006;13(6):422-426. doi:10.1016/j.knee.2006.08.003

16. Mumingjiang Y, Zhou X, He R. Value of knee skin temperature measured by infrared thermography and soluble intercellular adhesion molecule-1 in the diagnosis of peri-prosthetic knee infection in Chinese individuals following total knee arthroplasty. Chin Med $J$ (Engl). 2014;127(17):3105-3109.

17. Paton RW, Neal NC. Doppler assessment of blood flow to the lower limb associated with total knee arthroplasty. $J R$ Coll Surg Edinb. 1991;36(2):81-82.

18. Patil S, Allan DB, Quin R. Effect of total knee arthroplasty on blood flow to the lower limb: a prospective clinical study and review of literature. J Arthroplasty. 2002;17(7):882-886. doi:10.1054/arth.20 02.34536
19. Razani M, Parkhimchyk A, Tabatabaei N. Lock-in thermography using a cellphone attachment infrared camera. AIP Adv. 2018;8 (3):035305. doi:10.1063/1.5021601

20. Doesburg F, Smit JM, Paans W, Onrust M, Nijsten MW, Dieperink W. Use of infrared thermography in the detection of superficial phlebitis in adult intensive care unit patients: a prospective single-center observational study. PLoS One. 2019;14(3):e0213754. doi:10.1371/journal.pone.0213754

21. Owen R, Ramlakhan S. Infrared thermography in paediatrics: a narrative review of clinical use. BMJ Paediatr Open. 2017;1(1): e000080. doi:10.1136/bmjpo-2017-000080

22. Knobel-Dail RB, Holditch-Davis D, Sloane R, Guenther BD, Katz LM. Body temperature in premature infants during the first week of life: exploration using infrared thermal imaging. $J$ Therm Biol. 2017;69:118-123. doi:10.1016/j.jtherbio.2017.06.005

23. Samadi N, Thapa D, Salimi M, Parkhimchyk A, Tabatabaei N. Lowcost active thermography using cellphone infrared cameras: from early detection of dental caries to quantification of THC in oral fluid. Sci Rep. 2020;10(1):7857. doi:10.1038/s41598-020-64796-6

24. Kanazawa T, Nakagami G, Goto T, et al. Use of smartphone attached mobile thermography assessing subclinical inflammation: a pilot study. J Wound Care. 2016;25(4):177-182. doi:10.12968/ jowc.2016.25.4.177

25. Mu J, Liu D, Ji D, et al. Determination of pneumatic tourniquet pressure of lower limb by ultrasonic doppler. Ann Plast Surg. 2018;80(3):290-292. doi:10.1097/SAP.0000000000001247
Medical Devices: Evidence and Research

\section{Publish your work in this journal}

Medical Devices: Evidence and Research is an international, peerreviewed, open access journal that focuses on the evidence, technology, research, and expert opinion supporting the use and application of medical devices in the diagnosis, monitoring, treatment and management of clinical conditions and physiological processes. The identification of novel devices and optimal use of existing devices which will lead to improved clinical outcomes and more effective patient management and safety is a key feature of the journal. The manuscript management system is completely online and includes a very quick and fair peer-review system. Visit http:// www.dovepress.com/testimonials.php to read real quotes from published authors. 